(C) 1983 IEEE. Personal use of this material is permitted. However, permission to reprint/republish this material

for advertising or promotional purposes or for creating new collective works for resale or redistribution to servers or lists, or to reuse any copyrighted component of this work in other works must be obtained from the IEEE.

\title{
A High Current Injector for the Boeing Radiation Laboratory FEL Experiment
}

J. L. Adamski, W. J. Gallagher, R. C. Kennedy and A. D. Yeremian Boeing Aerospace Company, Seattle, Washington

\section{Summary}

A two-stage subharmonic injector has been designed and installed to accelerate 150 ampere micropulses with the Boeing $20 \mathrm{MeV}$, S-band linac. The accelerator will provide microbunches with interpulse time of 50 nanoseconds throughout the RF macropulse of 20 microseconds.

The electron source is a $5 \mathrm{~cm}^{2}$ triode electron gun which is gated to produce $5 \mathrm{~A}, 3$ ns pulses. Two standing wave prebuncher cavities are used, the first at $119 \mathrm{MHz}$, the 24th subharmonic of the linac frequency, and the second at 476 $\mathrm{MHz}$, the sixth subharmonic. A short tapered phase velocity traveling wave structure provides the final bunching. The solenoidal containment field is adjustable for optimization using injector profile diagnostics.

Computer analysis of bunching and radial trajectories predict beams of 2.2 nanocouloumbs with radius of $0.2 \mathrm{~cm}$ and phase width of $\pi / 2$ of the fundamental at the entrance to the traveling wave section.

\section{FEL Experiment Requirements}

The Boeing linac is a three section, traveling wave, disk-loaded structure powered by a single $20 \mathrm{MW}$ S-band klystron. The accelerator provides electron beams at $20 \mathrm{MeV}$ and $0.2 \mathrm{~A}$ for the MSNW/Boeing tapered wiggler free electron laser amplifier experiment. Conversion of the experiment to a FEL oscillator will require peak micropulse current in excess of $100 \mathrm{~A}$ for optical gain. The micropulse separation time is set equal to the two-way oscillator cavity transit time to maintain overlap of the electron pulses with the optical pulse. The total pulse train must be 20-100 microseconds to assure measurement of saturated gain and oscillator stability. Beam emittance $\varepsilon_{n}<0.01 \pi \mathrm{m}_{\mathrm{o}} \mathrm{c}-\mathrm{cm}$ and energy spread of $\triangle E / E<0.02$ represent allowable detuning within the FEL trapping potential well.

\section{Physical Description}

The subharmonic injector, Figure 1 , consists of a $10 \mathrm{~A}$, $100 \mathrm{kV}$ triode electron gun, two standing wave cavity prebunchers, and a fundamental frequency traveling wave tapered phase velocity buncher. A pepper pot emittance measurement and tuning diagnostics occupy the space between the prebuncher cavities. A full solenoidal magnetic field provides radial containment and focusing of the electron beam. A tapered collimator in the last drift section limits beam size and entry angle at the buncher. The injector components are described in the following sections.

\section{Electron Gun}

The Model 12B electron source is a gridded gun with 5 $\mathrm{cm}^{2}$ scandate dispenser cathode. The Model $12 \mathrm{~B}$ is a derivative of the original ARCO/RPC Model 12 with reduced cathode area. Simulation calculations using the SLAC EPT codel predicted a factor of 10 improvement in emittance for the small cathode version. The ETP calculation, Figure 2, predicts an output current of $10.7 \mathrm{~A}$ at $100 \mathrm{kV}$ with emittance of $3.1 \times 10^{-3} \pi \mathrm{moc}-\mathrm{cm}$.

A hard tube amplifier circuit provides the $1 \mathrm{kV}$ cathode drive pulse for $5 \mathrm{~A}$ with 3 ns FWHM. The cathode pulse circuitry will be phase locked to the master oscillator to provide electron pulses every sixth cycle of the first prebuncher ( 50.4 ns separation).

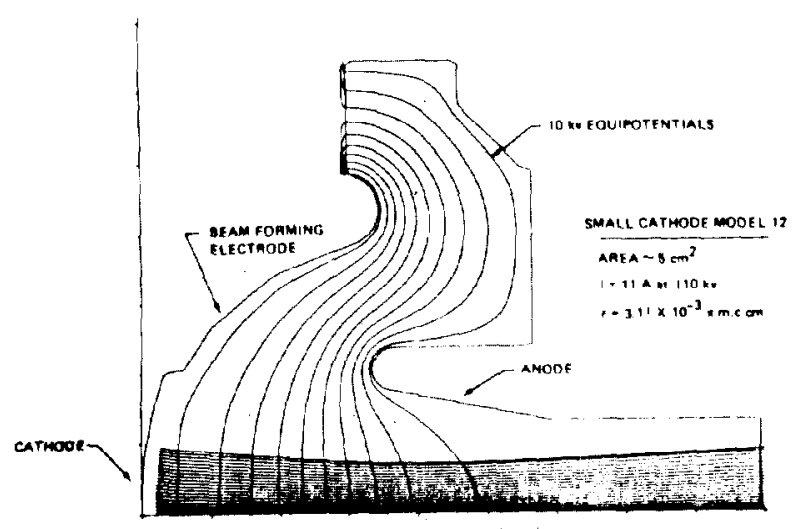

Figure 2. ETP Code Plot of Electron Trajectories for Modified Model 12 Gun

\section{Subharmonic Prebunchers}

Two-stage subharmonic bunching is used in a manner similar to the SLC injector. ${ }^{2}$ The prebuncher frequencies are chosen so that the electron pulse input phase width is roughly $+60^{\circ}$ in each of the cavities. The electron beam is then compressed by the relatively linear portion of the sinusoidal waveform and we are able to take advantage of nearly the full gap voltage.

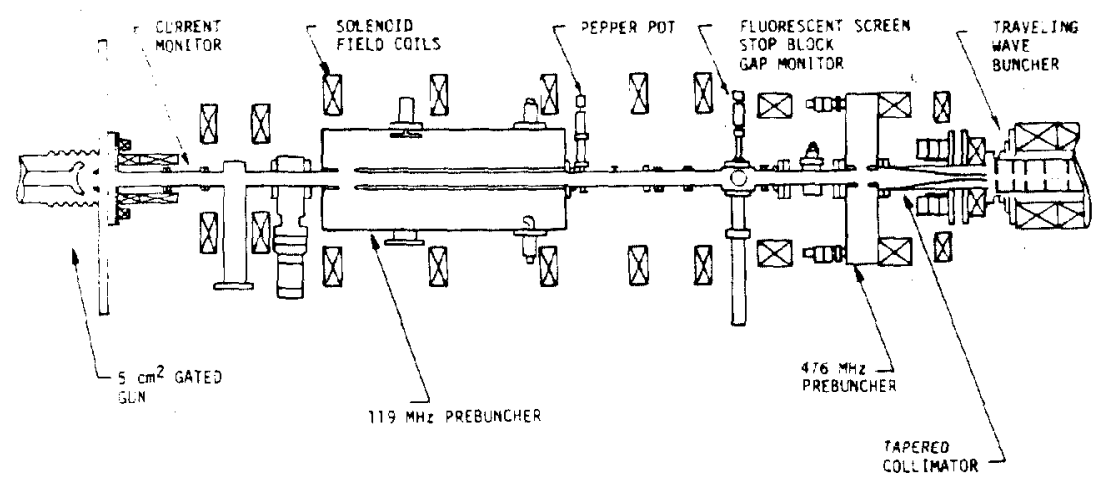

Figure 1. Two-Stage Subharmonic Injector 
The first prebuncher is a copper quarter-wave coaxial cavity. It operates at $119 \mathrm{MHz}$, the 24 th subharmonic of the accelerating frequency. The electrical properties are as follows:

$$
\begin{aligned}
& \mathrm{R} / \mathrm{Q}=305 \Omega \\
& \mathrm{Q}_{\mathrm{O}}=9900 \\
& \mathrm{Q}_{\mathrm{L}}=5400
\end{aligned}
$$

Operating point $=30 \mathrm{kV}$ gap voltage

Power requirement $=270 \mathrm{~W}$

The second prebuncher is a stainless steel pancake cavity operating at $476 \mathrm{MHz}$, the sixth subharmonic frequency. The electrical properties are:

$$
\begin{aligned}
& \mathrm{R} / \mathrm{Q}=30.7 \Omega \\
& \mathrm{Q}_{\mathrm{O}}=2263 \\
& \mathrm{Q}_{\mathrm{L}}=1340
\end{aligned}
$$

Operating point $=20 \mathrm{kV}$ gap voltage

Power requirement $=4.9 \mathrm{~kW}$

\section{Bunching}

The interaction of gated gun pulses with the standing wave cavities has been analyzed using the code ORBIT.3,4 The calculation considers the beam as a series of charged disks, ascribing all the beam charge to $\mathrm{N}$ disks per wavelength. The potential inside the beam pipe may be obtained, which conveniently eliminates infinities which would otherwise occur as disks approach each other infinitisimally close. The force on a disk may then be determined by summing over all other disks, and the disk motion calculated. The model is realistic in that it permits crossovers.

The code was used to model the cavities separately, and in combination. The input current was divided into 21 disks, each disk was assigned a charge so that the assembly represents a gaussian waveform containing 2.5 nanocoulombs with a pulse width of three nanoseconds. The computer output, Figure 3, plots relative, axial disk position in units of fundamental accelerator phase. Phase focussing, or bunching is exhibited since at any location, $z$, the isochronous condition is along a line whose slope is

$\frac{d \phi}{d z}=\frac{2 \pi}{8 \lambda}$

$\begin{aligned} \phi . \lambda & =\text { fundamental phase and wavelength } \\ B C & =\text { gun electron velocity. }\end{aligned}$

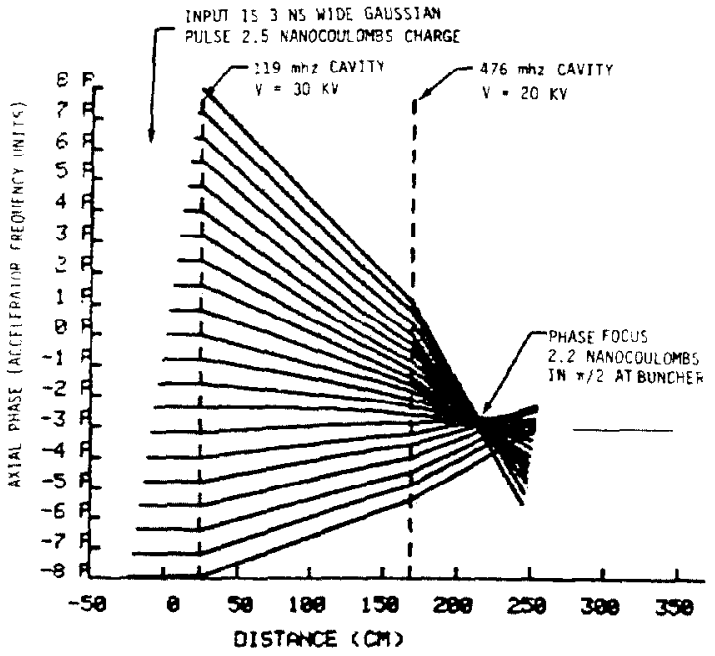

Figure 3. Orbit Code Bunching Plot for 2-Stage Subharmonic Injector.
In Figure 3, the $119 \mathrm{MHz}$ cavity gap voltage is $30 \mathrm{kV}$ and the $476 \mathrm{MHz}$ cavity voltage is $20 \mathrm{kV}$. Ninety percent of the input charge is focused to a fundamental phase width of $\pi / 2$ at $220 \mathrm{~cm}$, the entrance location of the tapered phase buncher. Additional bunching in this section will bring the final pulse width to 15-18 degrees of phase. The equivalent peak micropulse current is $150-170$ amperes.

\section{Magnetic Optics}

The gun output optics is comprised of a two section, 4 $\mathrm{cm}$ radius solenoid inside a short, $8 \mathrm{~cm}$ radius reverse current coil. The reverse coil bucks out field in the cathode-anode gap and allows quick transition into the uniform axial field region. This transition region was modeled by ETP. The results, in Figure 4, are compared with conventional optics using steel-jacketed lens outside the Model 12B gun. The solenoid output optics displayed a more laminar flow with no radial crossovers. Both lens systems focused to the same final radius. The calculated output emittance was a factor of three lower for the solenoid optics.

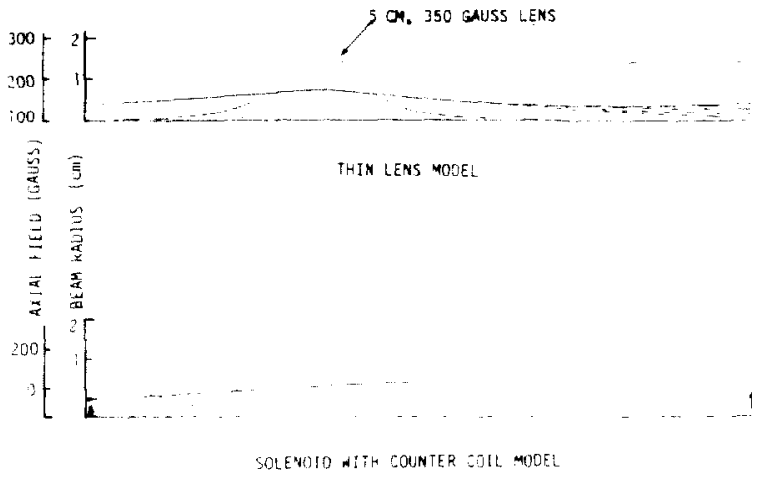

Figure 4. ETP Code Plots Compare Designs For Electron Gun Output Optics

The main solenoid field ranges from 200-400 gauss between the prebunchers to 2500 gauss at the entry to the buncher. The injector magnetic optics is modeled with a minicomputer code LENSING. The code performs a paraxial ray analysis to calculate beam envelopes in the injector. Beam current ramping by the prebunchers is included. The code has been directly compared with ETP for the solenoid optics case in Figure 4. Agreement in the calculation of beam radii was within $10 \%$ throughout the full axial distance. The code also adjusts injector coil currents to fit a Brillouin flow magnetic field profile.

A calculation of the composite magnetic field and the beam envelope is shown in Figure 5. The gun current is $3 \mathrm{~A}$. Each of the prebunchers linearly increases the current by a factor of 4. The beam radius is $1 \mathrm{~cm}$ at the gun, tapering to $0.2 \mathrm{~cm}$ at the fundamental buncher input. It is observed in the calculations that a slight mistuning of the coils gives rise to large amplitude "coke bottle" radial oscillations. To facilitate the expected experimental optimization, 12 separate programmable circuits are used to excite the solenoid field. A CAMAC/minicomputer system will be used to calculate axial field and to reset specific field profiles.

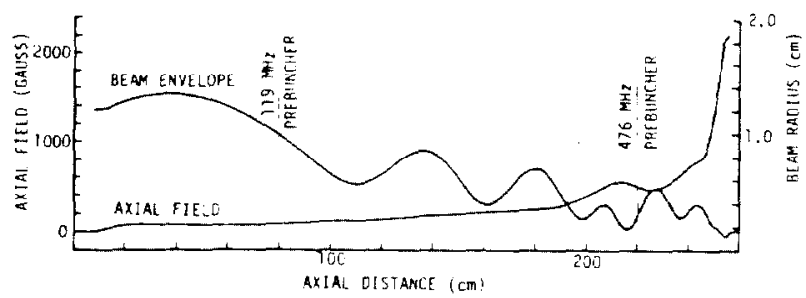

Figurc 5. LENSING Code Plot of Injector Magnetic Field and Electron Beam Envelope 
Primary tuning diagnostics include two fast toroid current monitors and a combination stopping blockfluorescent target. In addition, an emittance diagnostic is built into the drift space between the two prebunchers. A multiple hole collimator (pepper pot) can be inserted $40 \mathrm{~cm}$ upstream of the fluorescent target. In a constant axial field $B_{z}$, a waist of radius $r_{0}$ is set at the collimator (evidenced by no overall magnification of the collimator pattern at the screen). If the size of the beamlets from the collimator holes is dominated by rotation in the axial field, then the individual collimator hole image radius, $r^{\prime}$, is a function of $\alpha$, the beam far-field angle, and the solenoid field. The emittance can be estimated as follows:

$\varepsilon=\pi r_{0} \alpha=\pi r_{0} \frac{e}{m_{0} c} \frac{B_{z} r^{\prime}}{B r}$.

\section{Acknowledgements}

The contributions of E. L. Tyson, J. R. Beymer, C. P. Baze and R. A. Zilbert to the design and construction of the injector are greatly appreciated. We would also like to acknowledge the direction and support of D. R. Shoffstall.

\section{References}

1. W. B. Hermannsfeldt, "Electron Trajectory Program," SLAC Report 226, November 1979.

2. M. B. James and R. H. Miller, "A High Current Injector for the Proposed SLAC Linear Collider," 1981 Particle Accelerator Conference, Wash., DC, March 11-13, 1981, p. 3461.

3. G. W. Peterson and W. J. Grallagher, IEEE Trans. Nucl. Sci. NS-16, 214 (1969).

4. G. Mavrogenes, et al., "Subnanosecond High Intensity Beam Pulse," IEEE Trans. Nucl. Sci. NS-20, 919 (1973). 